\title{
Fuzzy Logic Approach for Cluster-Head Election in Wireless Sensor Network
}

\author{
Safana H. Abbas ${ }^{1}$ and Israa M. Khanjar ${ }^{2}$ \\ ${ }^{1}$ Professor, Al-Mustansiriya University, Department of Computer Science, Baghdad, Iraq \\ ${ }^{2}$ Research Scholar, Al-Mustansiriya University, Department of Computer Science, Baghdad, Iraq
}

\begin{abstract}
Wireless Sensor Network (WSN) consists of small battery-powered sensor nodes with limited energy resources, with sensing, computation, and communications capabilities. One of the crucial issues in WSN is energy consumption thus, poor energy efficient routing. The lifetime of the entire network depending on the energy efficiency of the sensor network which finally requires an energy-efficient routing protocol. This paper provides energy-efficient routing by using a fuzzy logic approach in the clusterhead selection process that provides a completely non-probabilistic approach in order to balance the energy consumption of nodes and prolong the WSN lifetime. This approach uses three fuzzy variables: Residual energy of the nodes, distance to Base station and distance to the cluster-head. Simulations results show that the proposed algorithm improves energy consumption and significantly prolong the network lifetime in wireless sensor networks when compared with LEACH protocol.
\end{abstract}

Key Words: Cluster head, Fuzzy logic, Energy, Routing, Wireless Sensor Network.

\section{INTRODUCTION}

Wireless Sensor Network is one of the most common areas of research in the networking field due to the continuous improvement in the fields of wireless technology and embedded system [1]. It consists of a group of small sensor nodes in an ad hoc mode capable to communicate wirelessly with sensing, computation and communications capabilities [2, 3]. Thees sensors trace specific environment phenomenon and implement in-network processing on the traced data before passing the results to the main aggregation device, called the sink or Base Station and then to the user through the internet [4].

WSNs are self-organizing networks used for monitoring the physical world entities such as temperature, pressure, light, moisture, sound etc. [5]. Since the sensor nodes are battery-powering and may be deployed in an inaccessible and dangerous environment, it is difficult and impractical to recharge or replace their power resources. Therefore, optimizing energy consumption is a critical problem in WSN. Hence, efficient-routing technique is very required to extend the lifetime of network and balance energy consumption between nodes in the network $[6,7]$.

Base station has unconstrained energy and can communicate with the end-user over the Internet, satellites or any communication medium. In fact, the cluster-head act as a gateway between base station and the nodes. The optimal formation of clusters and the suitable cluster-heads selection plays a main role in decreasing energy consumption [7].

Clustering is one of the efficient techniques [8], and the proper cluster-head selection can significantly reduce the consumption and extend the life time of WSN. In clustering protocols, cluster-head behave like a local coordinator for its cluster and responsible for many actions [9]. The efficiency of clustering techniques in increasing the lifetime of network has been proven in many studies, like the Low Energy Adaptive Clustering Hierarchy protocol (LEACH) and its improvements. The clustering technique is considered to be energy efficient when its execution is managed well in terms of cluster formation and the selection criteria of cluster-heads in the network [10].

In general neural network, genetic algorithm, fuzzy logic, evolutionary algorithm, swarm intelligence, and reinforcement learning are different algorithms used in WSNs. These algorithms could be truly utilize the distributive characteristics of WSN and significantly reduce the communications overheads [11]. In this paper, the proposed algorithm uses Fuzzy logic approach to realize the efficient routing algorithm for wireless sensor network.

\section{RELATED WORK}

Routing protocol design is one of the most active research fields that contribute in network life prolongation [12]. When it comes to effective routing and the network lifetime, hierarchical or cluster based routing is the better choice [10]. In the last years, 
many researchers have studied the routing and energy consumption problem in WSNs. There are many intelligent methods used in routing researches of wireless sensor network such as fuzzy logic. In this section, a survey for several protocols that use fuzzy logic is presented. In ref. [13] the authors present a fuzzy clustering algorithm which improves the energy efficiency of LEACH protocol by using a better cluster head selection solution. For this propose, they changed the probabilistic cluster-head selection in the LEACH protocol and apply the number of live neighboring nodes and remaining energy of network nodes to select better nodes for clustering. This scheme reduces the total node distance to cluster-head. This finally, reduces the energy consumption of sensors and improves the WSN lifetime better than LEACH protocol.

In [14], they proposed a novel energy efficient system using fuzzy logic with three parameters comprising the energy amount of $\mathrm{CH}$, the distance from cluster-head to base station and the number of connections in CHs. In fact, they focused on the clusterformation process and compared the proposed protocol with LEACH and FLCFP algorithms. The simulation results showed 2 12\% improvement in lifetime of network compared with LEACH and FLCFP.

In paper [15] the authors proposed an improvement of WSN clustering using fuzzy logic approach. Remaining energy, distance to the sink, and the density of nodes in their locality, were taken as the inputs into fuzzy inference system (FIS). A rank for each node is calculated for electing the cluster coordinator. Compared results with the other techniques such as LEACH, CAFL, and $\mathrm{CHEF}$ protocols showed the proposed system provided the better performance with regards to stability period and prolonged lifetime.

In [16], they proposed a fuzzy algorithm for cluster-head selection. Three parameters were taken into account: nodes residual energy, distance between nodes and mobile sink and the centrality of the cluster. This fuzzy method of selecting $\mathrm{CH}$ can balance the energy consumption of network well. Simulation results show that the proposed algorithm outperforms other common algorithms in terms of energy consumption and network lifetime.

In ref. [17], they designed a fuzzy logic scheme and proposed a clustering algorithm for cluster-head selection using fuzzy inference system (FIS) depend on remaining energy and RSSI to reduce energy consumption and prolong nodes lifetime. The proposed algorithm is compared with protocol LEACH. The simulation results display that the proposal performance better than LEACH in terms of energy consumption, nodes lifetime and number of sent data packets.

\section{ROUTING PROTOCOLS AND FUZZY LOGIC}

In this section, the most common hierarchal routing protocol is addressed and basic concepts of Fuzzy logic are presented.

\subsection{LEACH protocol}

LEACH is the most important, most popular, and widely used cluster based routing protocol for WSNs, which proposed by W. R. Heinzelman et.al, with the main aim of reducing power consumption [18, 19]. LEACH is the earliest distributed algorithm using a probabilistic threshold to elect cluster heads (CHs) [20]. LEACH working in a completely distributed manner and it does not need to know global knowledge of the network [18]. The major concept of this routing algorithm is to randomly rotate the cluster heads for each communication period among the different sensor nodes ( $\mathrm{SNs}$ ), so that the dissipated energy (which is required for a $\mathrm{SN}$ to be a $\mathrm{CH}$ ) is distributed among all of sensor nodes during the communication with the base station (BS) [19]. In LEACH, clusters are formed based on the Received Signal Strength (RSS) value for each SN and CHs are used as routers to the base station, as shown in Figure 1.

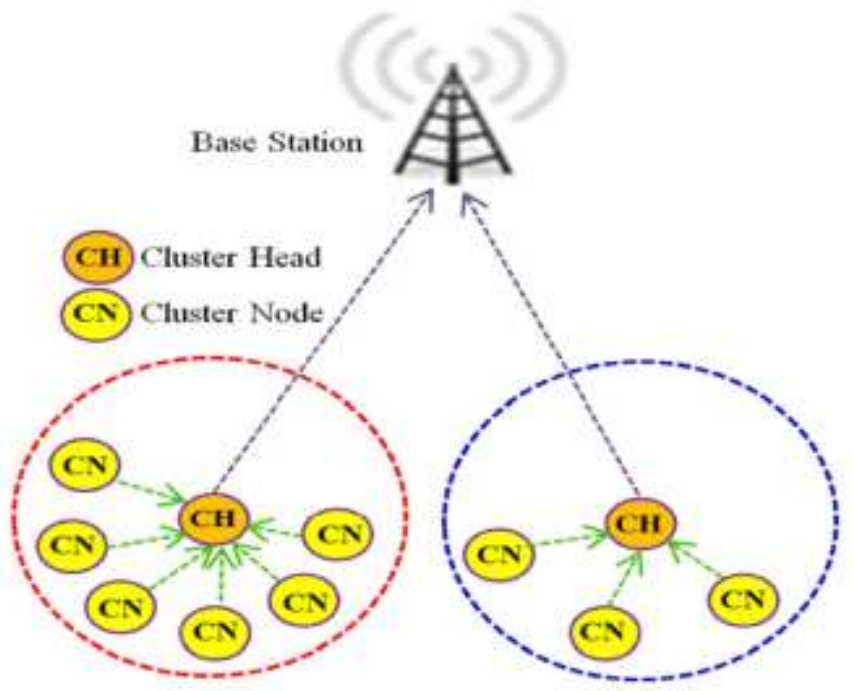

Figure 1: LEACH description [21] 
It was concluded after a number of simulations by authors that only $5 \%$ of overall number of sensor nodes will acting as clusterheads in the network. LEACH divides the entire sensor network into several clusters; each cluster has cluster-head and the network runtime is divided to rounds. Cluster head is a special node responsible for scheduling in TDMA (Time Division Multiple-Access) format. The other responsibility of $\mathrm{CH}$ is to aggregate the received sensed data and send the aggregated data to the base station and it is transmitted as a CDMA (Code Division Multiple Access) form in order to avoid the interference. LEACH used these two mechanisms to prevent collision; (TDMA) inside each cluster and (CDMA) between the clusters.

The implementing process of LEACH involves many rounds, each round has two phases: setup phase and steady- state phase as shown below:

- In setup-phase: the clusters are created and the CHs are selected randomly for every cluster.

- In steady-state phase: data is transmitting to the base station.

Figure 2 shows the illustration for the two phases, where the steady phase is divided into frames, and each sensor node routes its data to the cluster head one time per frame through its own time slot [22].

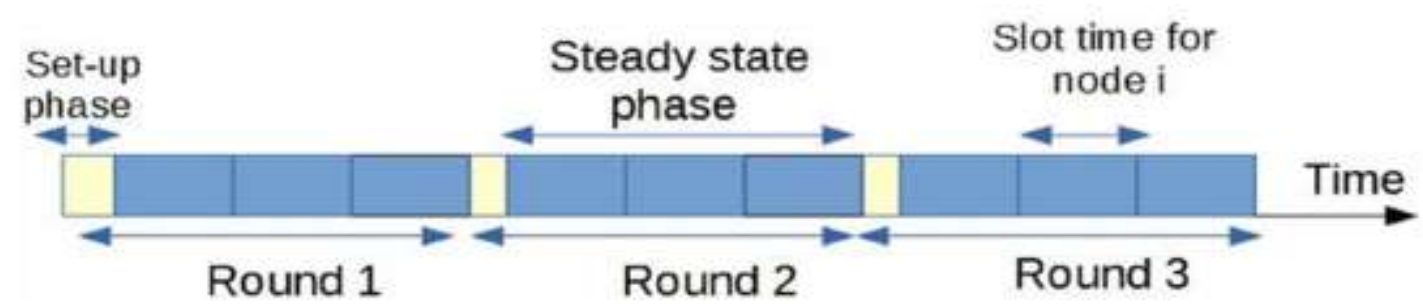

Figure 2: Time Division Multiple Access schedule for LEACH operation [23]

In the setup phase, the election of cluster-head depends on the threshold equation $\mathrm{T}(\mathrm{n})$. Each sensor node generates a random number between zero and 1, if this number of sensor node is less than the value of the threshold T(n), then the SN becomes a $\mathrm{CH}$ for this current round, else it can get its chance to be a cluster-head in the next rounds [20]. The T(n) is defined in (1):

$$
T(n)=\left\{\begin{array}{cc}
\frac{p}{1-p(r * \bmod (1 / p))} & \text { if } n \in G \\
0 \quad \text { otherwise }
\end{array}\right.
$$

Where:

P: is the Probability of selecting the $\mathrm{CHs}(\mathrm{P}=5 \%)$,

$r:$ is the current round

$\boldsymbol{n}:$ is the node

$\boldsymbol{G}$ : is the set of sensor nodes which have not been selected as CHs in the existing epoch. Epoch represents the no. of rounds which after it the node able to become $\mathrm{CH}$ again [23, 24].

Using that threshold, every SN will be a cluster head in some round within 1/p rounds. After the CHs are selected, they broadcast their status of being CHs using Carrier Sense Multiple Access (CSMA) Media Access Control (MAC) protocol. Each $\mathrm{SN}$ will determine the optimum cluster head to join based on the RSSI value provided by each $\mathrm{CH}$. Then, after the clusters are formed, the CHs build TDMA (Time Division Multiple Access) schedules depending on the no. of nodes in the clusters. Each node in these schedules is linked to a time-slot during which the node can send messages (the transmission is through the total bandwidth assigned for data transmission), meaning that each node trying to make the transmission through its own time slot, it is certain that the channel-free. Lastly in order to prevent interference of close clusters messages, each cluster head randomly chooses a code in the Code Division Multiple Access (CDMA) codes list and sends it to the nodes that belong to its cluster to be used during transmission. Therefore, all the nodes are using the same bandwidth but with the Frequency modulation that is determined in the codes sent by the cluster heads [22, 24].

In steady-phase, each sensor node forwards its data to the corresponding cluster head in its allocated time frame. Then, each $\mathrm{CH}$ collects the received data and aggregates it and then transfers the aggregated data to the BS [22].

\subsection{Fuzzy Logic Approach}

Fuzzy logic (FL) was first proposed by Lotfi-Zadeh and is used to model human behavior in decision making. FL imitates the logic of human thinking, which is much less rigid than the calculations normally made by computers. Fuzzy system provides many unique features which make it a good approach especially for several control issues. It has the ability to deal with ambiguity and uncertainty and allows the merging of multiple parameters, and often conflicting, in a single metric. In addition, Fuzzy logic has the advantages of, robustness, easy implementation, and ability to handle nonlinear systems [25]. 
Fuzzy logic system composed of four parts: a fuzzifier, the inference engine, Fuzzy rule-base and a defuzzifier. Figure 3 shows the block diagram of Fuzzy Inference System.

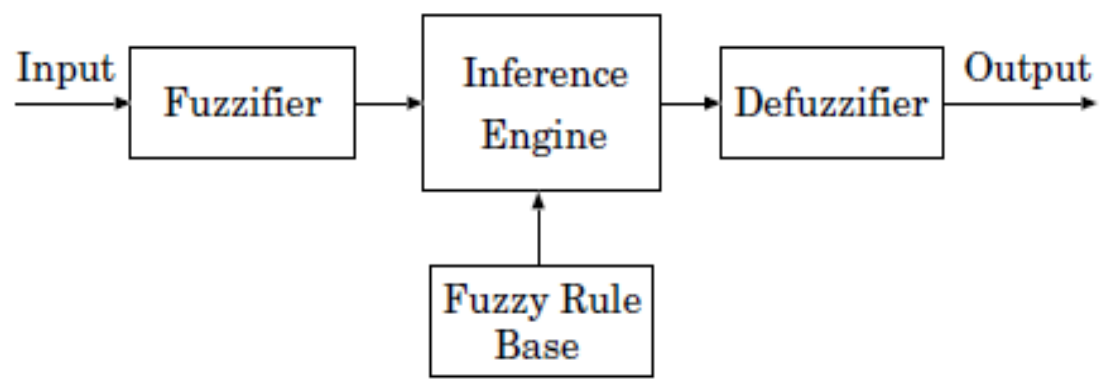

Figure 3: Basic structure of Fuzzy Logic System [26]

There are four required steps for completing the process:

1. Fuzzification: in fuzzification, the input is given with crisp values and converted to fuzzy sets. This can be done by fuzzifier.

2. Rules-evaluation: It is a store of IF-THEN rule.

3. Fuzzy Inference-Engine: the fuzzy inference engine (FIS) combines both the IF-THEN rules and input values to simulate the reasoning that results in a fuzzy inference.

4. Defuzzification: the Defuzzifier converts the fuzzy output into a crisp value [27].

\section{ROUTING THE PROPOSED FUZZY LOGIC (FL) BASED ROUTING APPROACH}

The major objective of the Proposed WSN Routing protocol is to extend the network lifetime. The proposal simulation is applied to a number of rounds similar to the LEACH protocol, each round consisting of the setup-phase and the steady state phase. Setup phase contains two steps: cluster formation, and cluster head selection. The selection process of the cluster head is done by Fuzzy Logic approach depending on different criteria. In this system three parameters are considered.

After the nodes are deployed, the sensor nodes must be divided into clusters. The first demand of the clustering is to choose $\mathrm{CH}$ of the cluster then its members. Therefore, the network includes two types of sensor nodes: Cluster-heads and normal nodes. After the TDMA scheduling, normal nodes for each cluster begin sending sensed data to the corresponding cluster heads which transmit aggregated data to the BS during the steady-phase.

\subsection{The Proposed algorithm}

The cluster head is elected by the Base Station using fuzzy logic approach which provides a more appropriate cluster-head election method. The fuzzy logic present completely non-probabilistic approach, it has been employed in the proposed protocol to enhance the cluster head selection process and creates more symmetric clusters which results in lower consumption of energy and prolong the WSN lifetime. The proposed fuzzy logic system is a Centralized algorithm performed by BS through some rounds. To determine the optimum cluster head, the algorithm used three parameters as inputs:

- The residual energy level of the nodes.

- Distance between the node and BS.

- The distance from the nodes to the current $\mathrm{CH}$.

Figure 4 illustrates the proposed FIS design for the $\mathrm{CH}$ selection.

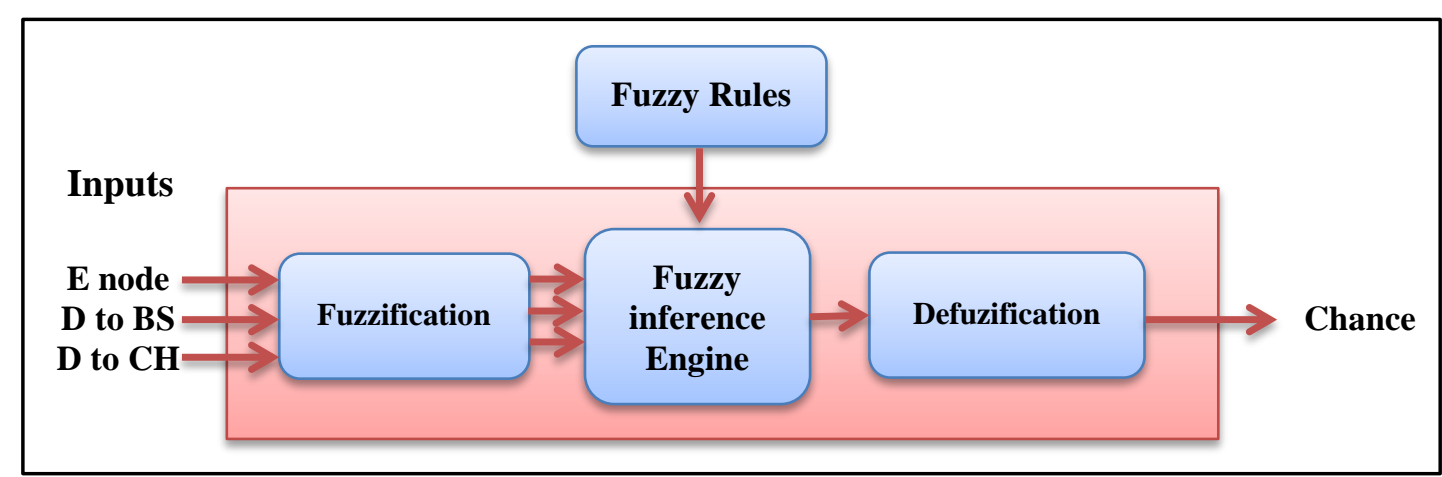

Figure 4: Block diagram of the fuzzy logic module for $\mathrm{CH}$-selection 
The output fuzzy value is the chance which represents the possibility of each node to be selected as cluster-head. After implementing the inputs parameters into FIS and calculating the chance of the nodes, the proposed routing protocol chooses the node with highest chance to be $\mathrm{CH}$ for next round. The figure 5 illustrates the flowchart of $\mathrm{CH}-\mathrm{Selection}$ process.

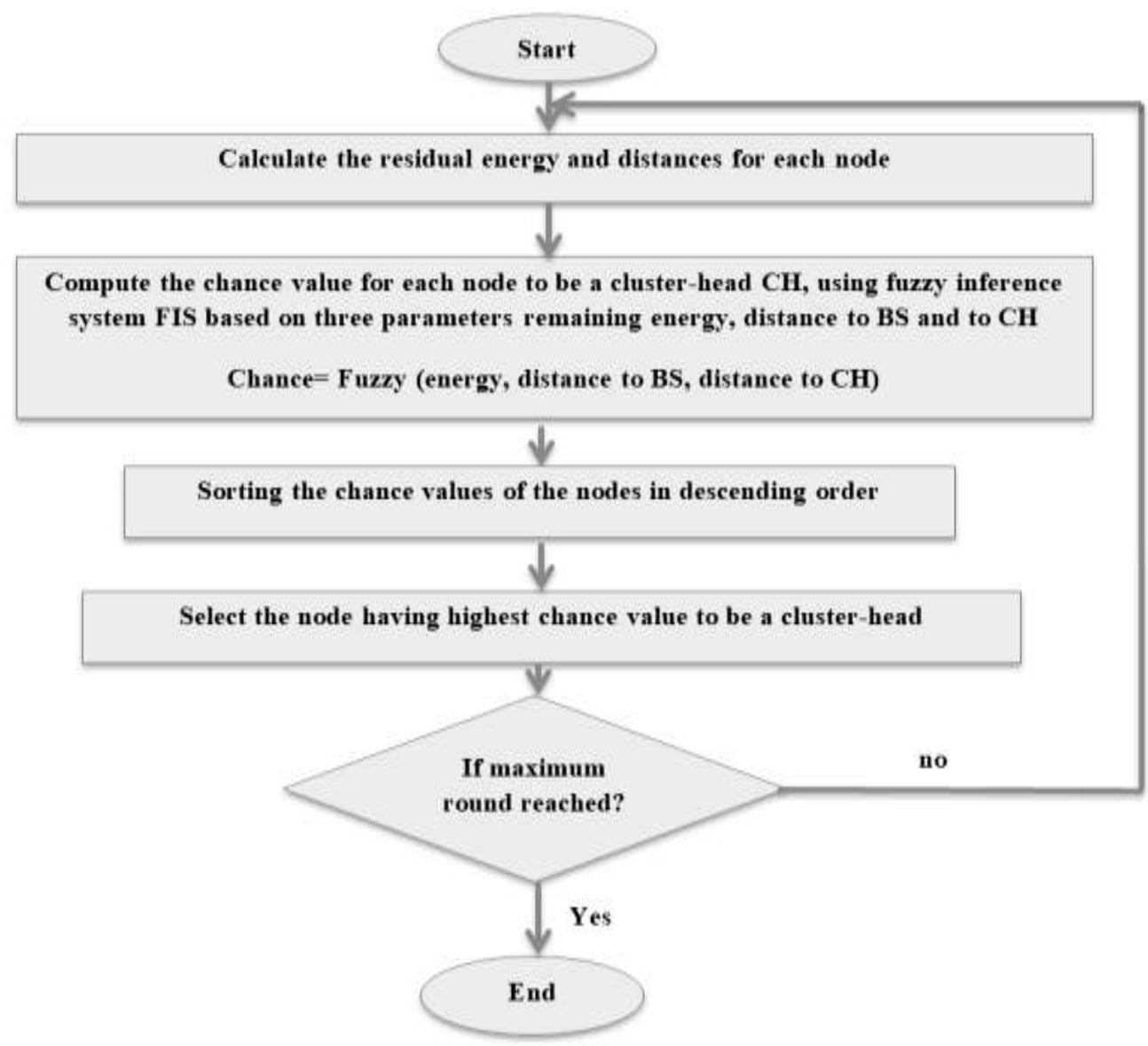

Figure 5: Flowchart of CH- selection

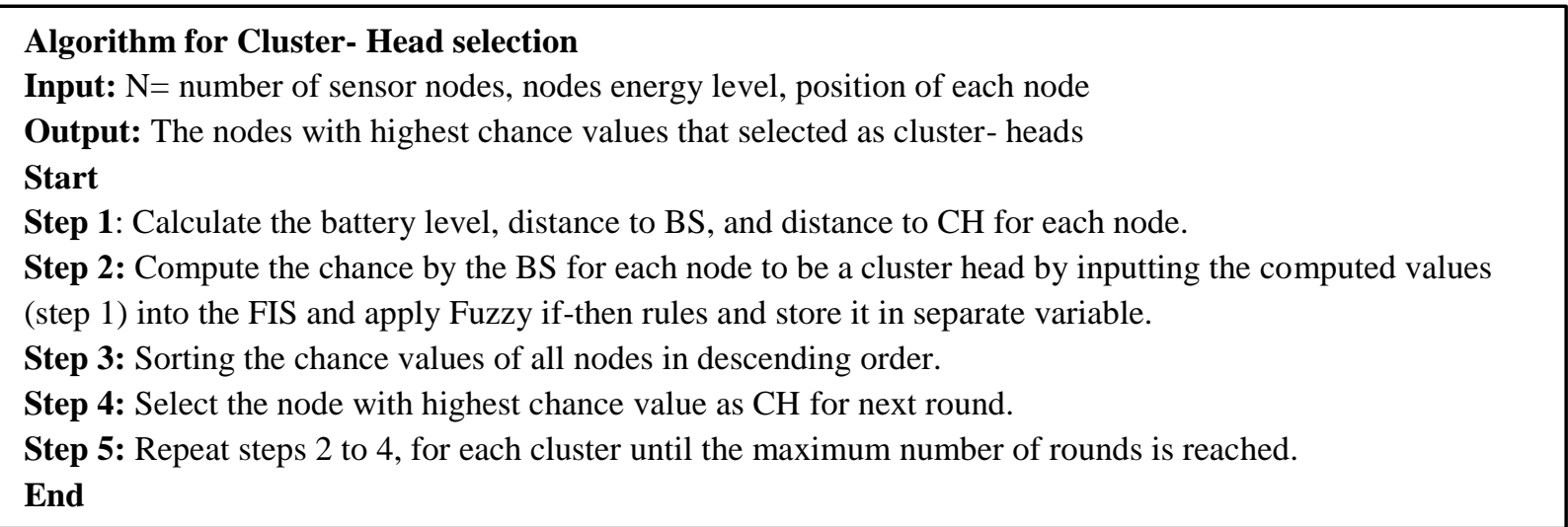

In the proposed algorithm, the cluster-head selection process is modified by providing an efficient $\mathrm{CH}$ replacement system. It is a condition in cluster head election for each next round. If the current cluster head does not consume a lot of energy during its round and has more energy than the required limit, the cluster head will remain $\mathrm{CH}$ for the next round too. This is how can save the energy consumed in routing the packets to determine the new $\mathrm{CH}$ and cluster formation. That is lead to balance the energy consumed of the nodes in the cluster and reduces the early death of the nodes. If the $\mathrm{CH}$ has energy less than the required limit, it will be replaced using the proposed fuzzy inference system.

The cluster head checks its remaining energy level periodically. If its value reaches the required energy level limit $E_{\text {limit }}$, the $\mathrm{CH}$ starts a new round to organize the cluster members again. The organization of a cluster is to elect a new $\mathrm{CH}$ using fuzzy inference system by the BS. If the remaining energy of the exiting $\mathrm{CH}$ exceeds energy level limit $E_{\text {limit }}$, each node preserves its role. The CHs send the TDMA schedule (Time Division Multiple Access) to their members to send the sensed data. 
The flowchart of the proposed cluster head role rotation scheme is presented in the figure 6:

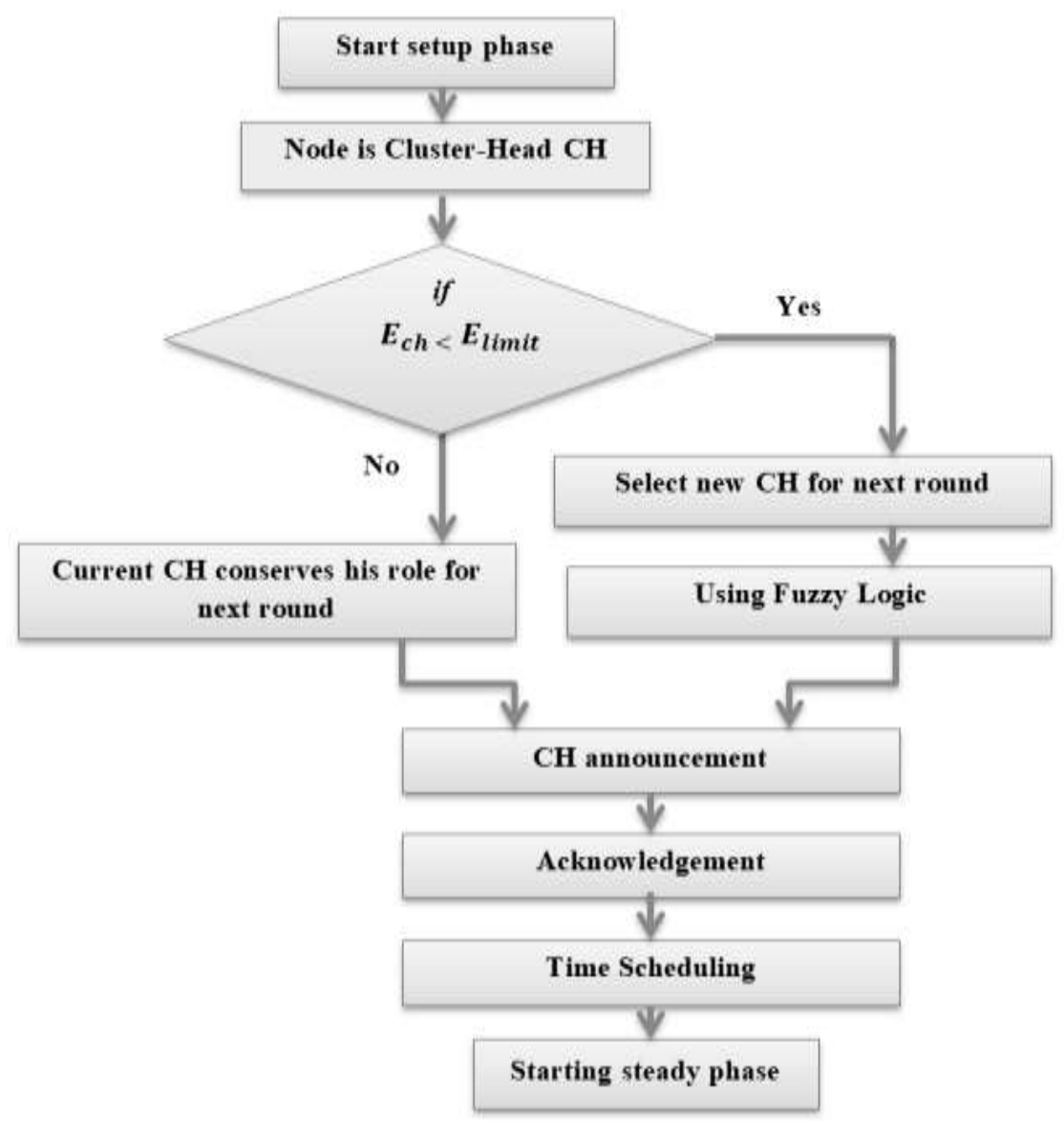

Figure 6: Flowchart of Cluster head replacement process for next rounds

\subsection{Designing Fuzzy inference system (FIS) model}

In the proposed system model, three parameters are considered: residual energy of the node, the distance from the node to the base station, and distance between the node and the $\mathrm{CH}$. These parameters are chosen according to their direct effect on energy dissipation and their importance in increasing the network lifetime. The output of FIS is defined as the chance, which determines the priority of the node to be cluster-head. Each linguistic variable used for representing these parameters is divided into three levels shown in table 1

Table1: Input functions

\begin{tabular}{|l|l|l|l|}
\hline Inputs & \multicolumn{3}{|c|}{ Membership Function } \\
\hline Node Energy & Low & Medium & High \\
\hline Distance to BS & Close & Medium & Far \\
\hline Distance to CH & Near & Medium & Far \\
\hline
\end{tabular}

Many membership functions types are presented in Fuzzy Logic toolbox of MATLAB comprising Trapezoidal, Triangle Gaussian, sigmoidal, Z-shape, and S-shape. On other hand, Trapezoidal and Triangle membership functions are more beneficial than other types. Therefore, they were selected for use in presenting the parameters as described in the following figures.

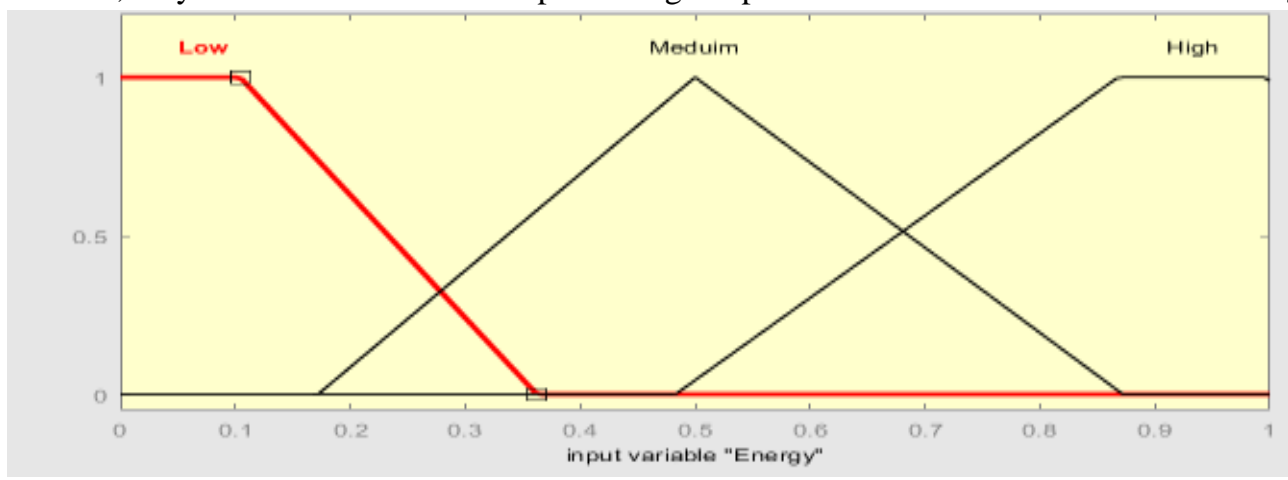

Figure 7: Fuzzy set of energy level 
International Journal of Engineering Research And Advanced Technology, Vol.5, Issue 7, July-2019

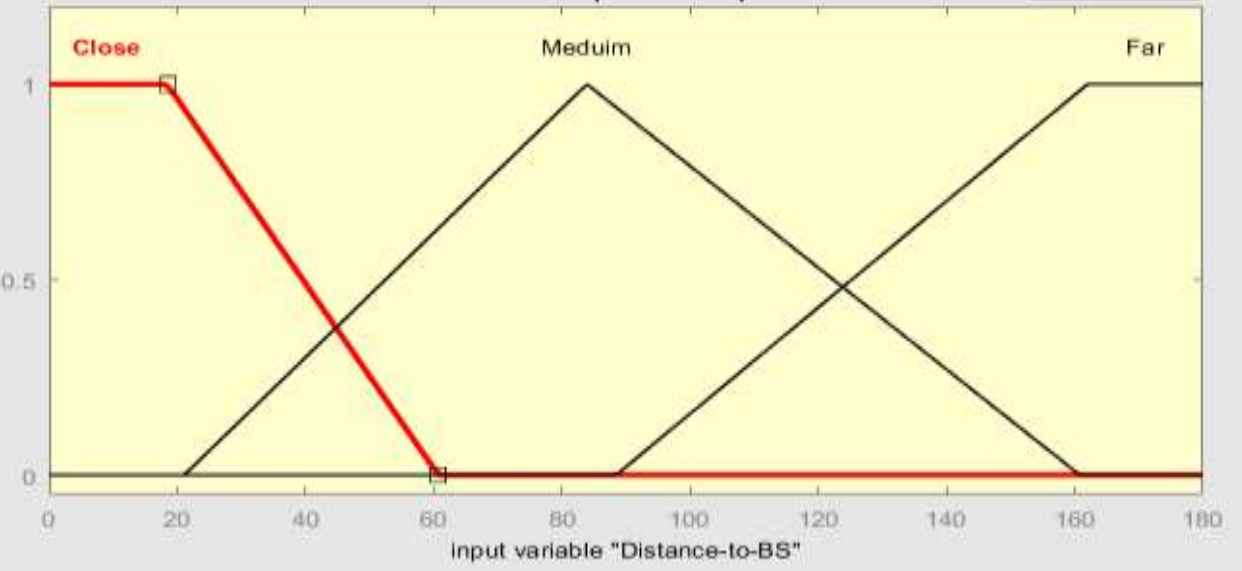

Figure 8: Fuzzy set of the distance to base station

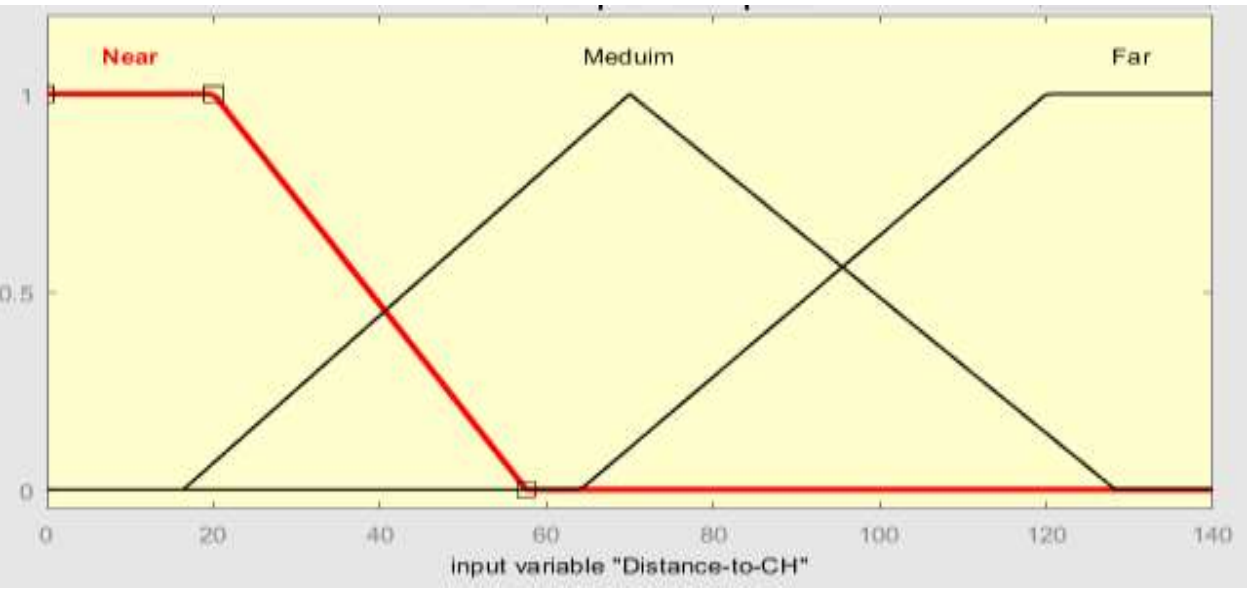

Figure 9: Fuzzy set of the distance to cluster head

The linguistic variable of the chance value was divided into nine levels as shown in table 2 :

Table 2: Output functions

\begin{tabular}{|c|l|}
\hline Outputs & \multicolumn{1}{|c|}{ Membership Function } \\
\hline Chance & $\begin{array}{l}\text { Very weak, weak, rather weak, rather } \\
\text { medium, medium, high medium, } \\
\text { rather strong, strong, very strong. }\end{array}$ \\
\hline
\end{tabular}

These chance levels represented by triangular and trapezoidal membership function as shown in Figure 10.

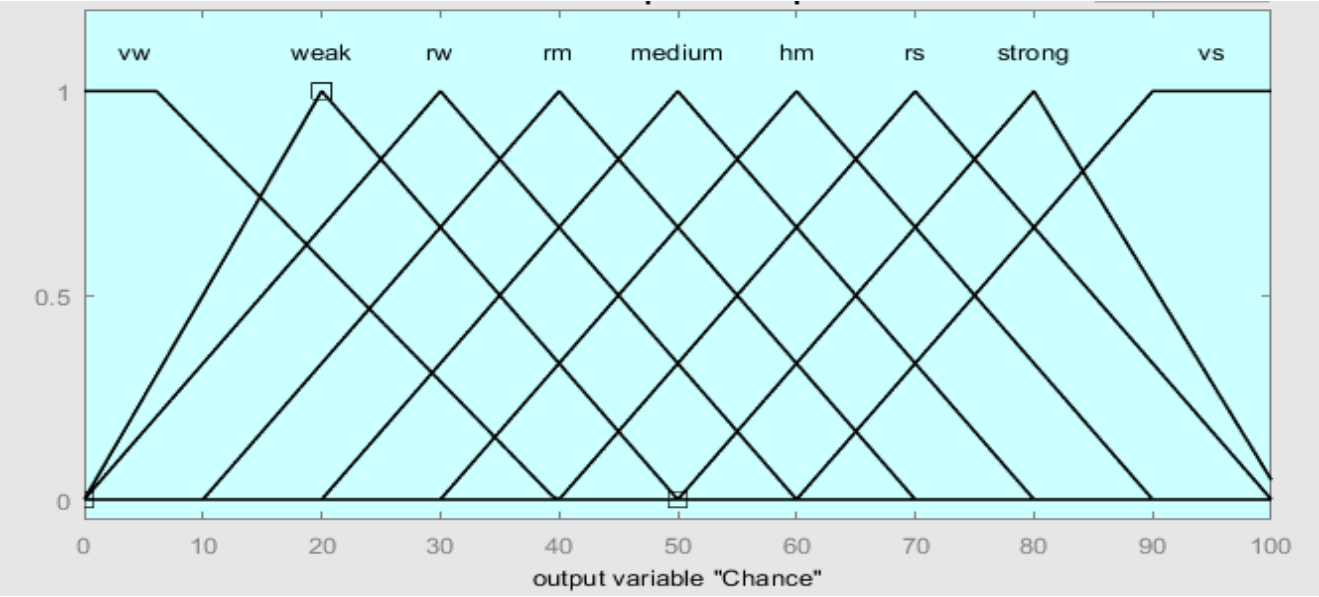

Figure 10: the chance value Fuzzy set

$\mathrm{vs}=$ very strong, $\mathrm{rs}=$ rather strong, $\mathbf{h m}=$ high medium, $\mathbf{r m}=$ rather medium, $\mathbf{r w}=$ rather weak, $v w=$ very weak 
International Journal of Engineering Research And Advanced Technology, Vol.5, Issue 7, July-2019

Each one parameter divided into three levels, there is $3^{3}=27$ possible chance values each corresponding to an IF-THEN rule shown in Table 3 below. These rules are located between two extremes cases as given below:

Case (1): If (Energy is low) and (Distance to BS is far) and (Distance to CH is far) then the (Chance is very weak) Case (2): If (Energy is high) and (Distance to BS is close) and (Distance to CH is near) then (Chance is very strong)

Table 3: IF-THEN rules for Fuzzy Inference System

\begin{tabular}{|c|c|c|c|}
\hline Energy & $\begin{array}{c}\text { Distance To } \\
\text { the BS }\end{array}$ & $\begin{array}{c}\text { Distance To } \\
\text { the CH }\end{array}$ & Chance \\
\hline Low & Far & Far & Very weak \\
\hline Low & Far & Medium & Weak \\
\hline Low & Far & Near & Rather weak \\
\hline Low & Medium & Far & Weak \\
\hline Low & Medium & Medium & Rather Weak \\
\hline Low & Medium & Near & Rather medium \\
\hline Low & Close & Far & Rather Weak \\
\hline Low & Close & Medium & Rather medium \\
\hline Low & Close & Near & Medium \\
\hline Medium & Far & Far & Rather weak \\
\hline Medium & Far & Medium & Rather medium \\
\hline Medium & Far & Near & Medium \\
\hline Medium & Medium & Far & Rather medium \\
\hline Medium & Medium & Medium & Medium \\
\hline Medium & Medium & Near & High medium \\
\hline Medium & Close & Far & Medium \\
\hline Medium & Close & Medium & High medium \\
\hline Medium & Close & Near & Rather strong \\
\hline High & Far & Far & Medium \\
\hline High & Far & Medium & High medium \\
\hline High & Far & Near & Rather strong \\
\hline High & Medium & Far & High medium \\
\hline High & Medium & Medium & Rather strong \\
\hline High & Medium & Near & Strong \\
\hline High & Close & Far & Rather strong \\
\hline High & Close & Medium & Strong \\
\hline High & Close & Near & Very strong \\
\hline
\end{tabular}

\section{Determining CH Chance Value:}

This part clarifies how to use the common fuzzy inference method called Mamdani. The next four steps present details for calculating the $\mathrm{CH}$ chance values in the FIS model.

\section{Step 1: Inputting the Crisp Values and Fuzzification}

Firstly, the crispy input values are forwarded to FIS model. Remaining energy of the node, distance to BS and distance to $\mathrm{CH}$. According to these 3 crisp inputs, the membership function values will determine.

\section{Step 2: Rule Evaluation}

when completing fuzzification step, and getting membership values, these values provided for the IF-THEN rule to identify new fuzzy outputs sets. The fuzzy operator (AND) is used for evaluating the rules to find the chance membership value.

\section{Step 3: Aggregating the Rules Output}

After fuzzification and rules evaluation is completed, aggregating step will begin. Aggregation is the operation of unifying all outputs resulted from implementing all rule (27 rule in this FIS model). 
In order to aggregate all the rules, the OR Fuzzy Logic operator has been used.

\section{Step 4: Defuzzification}

The last step in Fuzzy Inference system is Defuzzifaction where the $\mathrm{CH}$ election chance value is obtained. The (Centroid defuzzification) technique used to get a final crisp number, that representing the cluster head chance value for each node to be $\mathrm{CH}$.

Since the centroid is considered one of the most common defuzzification methods and it has been effectively implemented in various applications so, the Center of Area (COA) has been used in this work which can be computed by equation (2).

$$
\operatorname{COA}=\int f(x) * x d x / \int f(x) d x
$$

By apply the values obtained formerly from step 3 in the equation (2) and computing the algebraic integration, the value of chance for selecting the $\mathrm{CH}$ is determined about equals to (77\%) as illustrated in Figure 11 when Suppose that a node has energy level $(1 \mathrm{~J})$ and it is placed at a distance of $(10 \mathrm{~m})$ from base station, and the distance between this node and the previous cluster-head $(50 \mathrm{~m})$

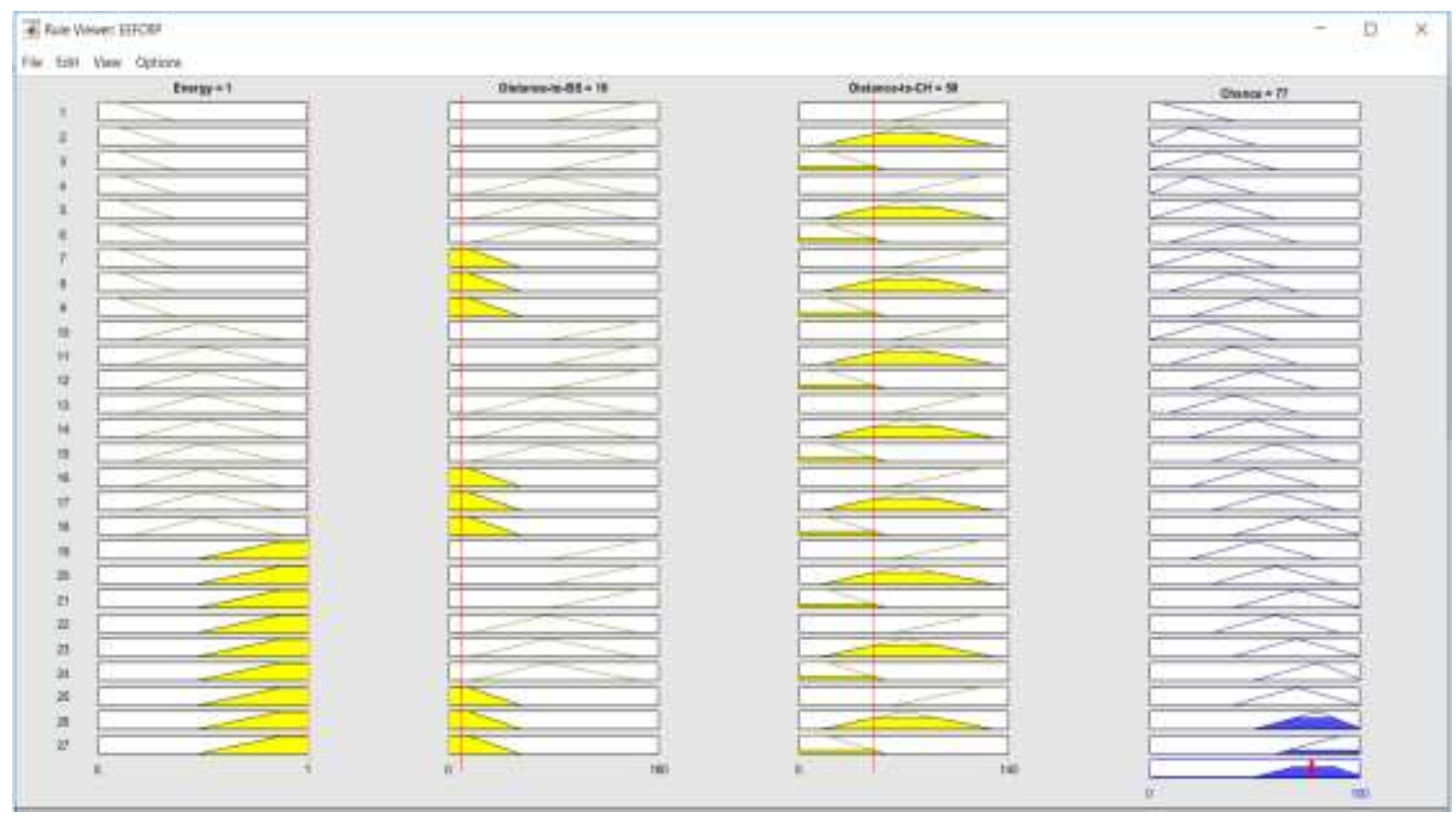

Figure 11: Evaluation output of fuzzy IF-THEN rules

Centroid defuzzification returns the (center of area) below the curve. The centroid COA is the point along $\mathrm{x}$-axis around it, that shape is balanced. Although, methods other than the centroid can be used to calculate $\mathrm{CHs}$ chance, but centroid is further suitable and easier than others as mentioned earlier.

\section{SIMULATION SETUP AND RESULTS}

In this section, the simulation of the proposed algorithm is presented in the environment of the network and compared to the LEACH protocol in the WSN. System modeling and simulation are made under MATLAB (R2017 b) using a windows 10 OS on an i7 PC. The simulation is accomplished using n-sensor nodes distributed randomly inside a $100 \times 100$ meter area. Simulation runs with the listed parameters in Table (4).

Table (4): The parameters of simulation 


\begin{tabular}{|c|c|}
\hline Parameters & Value \\
\hline Network size & $100 \mathrm{~m}^{2}$ \\
\hline Number of nodes & 100 \\
\hline Number of sinks & 1 \\
\hline Base Station location & $(50,150)$ \\
\hline E $_{0}=$ Initial energy for each node & $1 \mathrm{~J}$ \\
\hline Elec $_{f s}$ & $50 \mathrm{~nJ} / \mathrm{bit}$ \\
\hline$\varepsilon_{f s p}$ & $10 \mathrm{pj} / \mathrm{bit} / \mathrm{m}^{2}$ \\
\hline$\varepsilon_{\mathrm{DA}}$ & $0.0013 \mathrm{pj} / \mathrm{bit} / \mathrm{m}^{4}$ \\
\hline
\end{tabular}

Due to the methodology of the algorithm proposed in this network, an appropriate $\mathrm{CH}$ will be used in every new round according to the selection process of the cluster head that relies on the higher node chance using the fuzzy logic approach, and also due to use the Energy Level threshold.

All this may improve the implementation performance of the proposed WSN algorithm from energy consumption and lifetime of the network to better than the LEACH protocol as follows.

\subsection{Lifetime of the Network}

The network lifetime of the proposed protocol and LEACH protocol in terms of live nodes and number of rounds is illustrated in Figure 12.

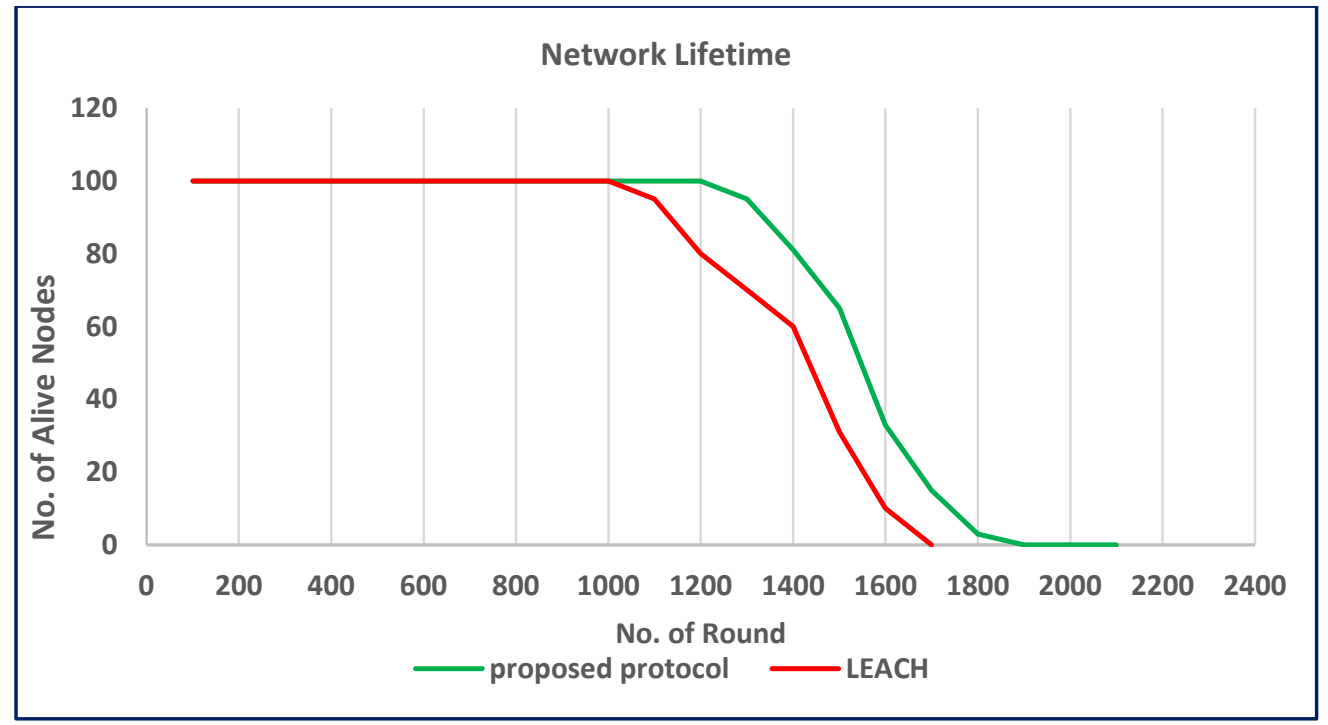

Figure12: Network Lifetime

Compared to $\mathrm{LEACH}$, the proposed protocol has an improvement during the stabilization period. As shown in figure 13, the first node die (FND) in the LEACH protocol in round 1002 and the last node died (LND) in round 1700. While In the proposed system, first node died is in round 1200 and the last node dies in round 1900.

The proposed protocol selects the best node as $(\mathrm{CH})$ that is changed depending on the maximum remaining energy and the minimum distance to the base-station and to the previous $\mathrm{CH}$ using the fuzzy logic which increases the network life time.

\subsection{Energy Consumption}

Minimizing energy dissipation is a significant problem for any routing protocol so that the network stays longer time for more rounds. Figure 13 illustrates the average energy consumption of all nodes in relation to the number of rounds. As shown the average energy dissipation in the proposed protocol is lower than in the LEACH protocol. The nodes in the proposed protocol exhaust their energy at a very slow rate compared to the LEACH protocol. 


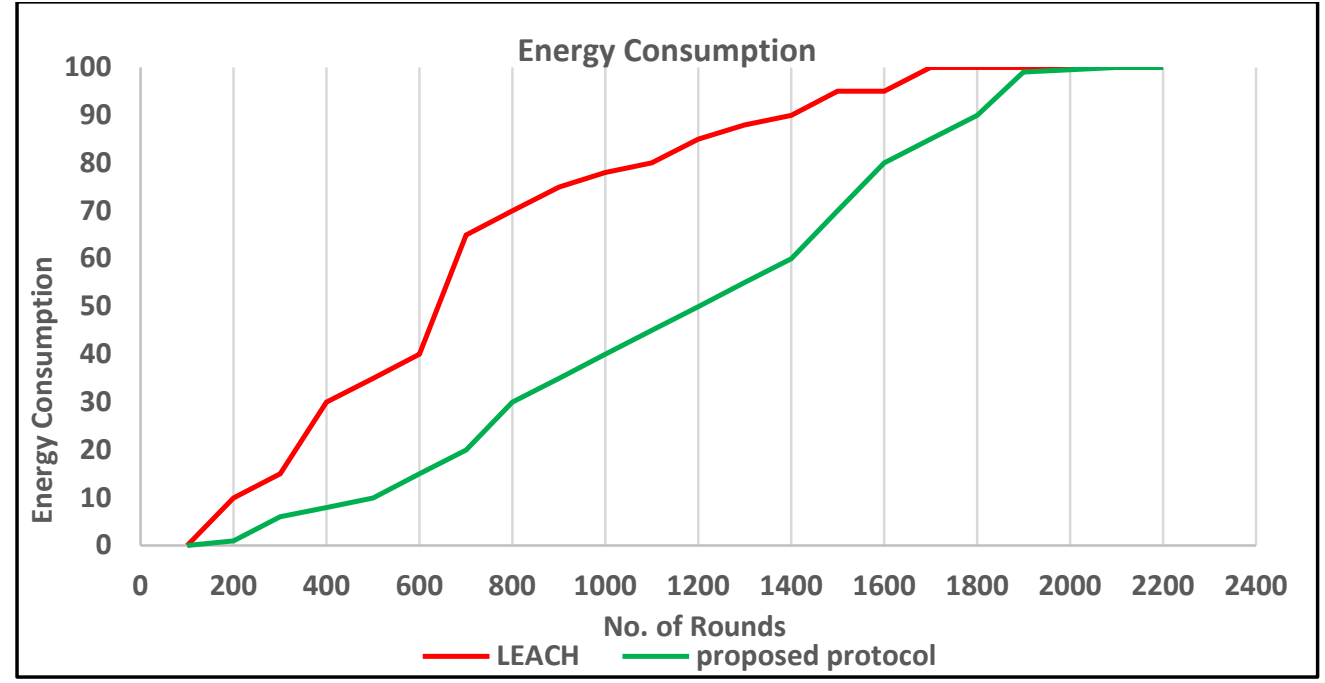

Figure (13): Energy Consumption of the Network

\section{CONCLUSIONS}

In WSN, it is very necessary to develop routing protocol that can preserve the nodes energy as much as possible to extend the network lifetime. The proposed algorithm uses Fuzzy inference system for routing in WSN. It utilizes the clustering method by selecting the cluster heads using fuzzy logic approach based on three parameters: node distance to the base station, distance to the cluster head, and the residual energy of the node. The proposed protocol is simulated and compared with LEACH protocol, from simulation results; some points are concluded from the performance of the proposed algorithm as the following:

- The proposed algorithm selects the Cluster-head near the cluster members with high remaining energy level using fuzzy logic approach, which results a chance value of the sensor node to determine which node is the best suited to be $\mathrm{CH}$ for total energy efficiency.

- By using three parameters to select CH by FIS, and using the Energy Level limit, the energy is dissipated in a balanced manner in the simulated network. Therefore, the proposed protocol does not suffer from the problem of an early death of nodes that may occur if some of the nodes consume higher energy rate than others.

- Different factors play a major role in choosing the cluster-head where only one factor cannot select the node as $\mathrm{CH}$ and also that the probabilistic concept does not guarantee the selection of cluster head well.

For future work, different parameters can be chosen in $\mathrm{CH}$ selection such as node density or centrality instead of one or more specified parameters. Selecting different criteria may more improve the performance of proposed work and there may be an opportunity to use artificial intelligence technique in the cluster head selection process.

\section{REFERENCES}

1. G.R. Asha and Gowrishankar, "Energy efficient clustering and routing in a wireless sensor networks." , Procedia computer science, India, Vol. 134, pp.178-185, 2018. [The 13th International Conf. on Future Networks and Communications (FNC 2018)]

2. S. Wang, J. Yu, M. Atiquzzaman, H. Chen, and L. Ni. "CRPD: a novel clustering routing protocol for dynamic wireless sensor networks., Personal and Ubiquitous Computing, Vol. 22, no. 3, London, 2018, pp.545-559.

3. S. Varshney, C. Kumar, and A. Swaroop. "A comparative study of hierarchical routing protocols in wireless sensor networks." In 2015 2nd International Conference on Computing for Sustainable Global Development (INDIA Com), IEEE, 2015, pp.1018-1023

4. H. M. Al-Mashhadi, "Simulation of Proposed Secure Method for wireless Sensor Network." M.SC thesis, University of Technology, Baghdad, Iraq, 2015.

5. K. Singh, and J. Kaur. "Machine Learning based Link Cost Estimation for Routing Optimization in Wireless Sensor Networks." Advances in Wireless and Mobile Communications, Vol. 10, Number 1: 0973-6972, pp. 39-49, 2017.

6. Z. M. Zahedi, R. Akbari, M. Shokouhifar, F. Safaei, and A. Jalali. "Swarm intelligence based fuzzy routing protocol for clustered wireless sensor networks." Expert Systems with Applications, Vol. 55 (2016): pp. 313-328.

7. S. Chelbi, M. Abdouli, M. Kaddes, C. Duvallet, and R. Bouaziz. "An Unequal Cluster-based Routing Protocol Based on Data Controlling for Wireless Sensor Network." International Journal of Electrical and Computer Engineering (IJECE), Vol. 6, No. 5, October 2016, pp. 2403 - 2414. 
8. A. Gummadi and K. RAO. "EECLA: Clustering and localization techniques to improve energy efficient routing in wireless sensor networks.” Journal of Theoretical \& Applied Information Technology, Vol.96, no. 1, pp. 204-216, 15th January 2018.

9. A. K. Mishra, R. Kumar, and J. Singh. "A review on fuzzy logic based clustering algorithms for wireless sensor networks." In International Conference on Futuristic Trends on Computational Analysis and Knowledge Management (ABLAZE), IEEE, pp. 489-494., 2015.

10. A. Messaoudi, R. Elkamel, A. Helali, and R. Bouallegue. "Distributed fuzzy logic based routing protocol for wireless sensor networks." In 2016 24th International Conference on Software, Telecommunications and Computer Networks (SoftCOM), IEEE, pp. 1-7, 2016.

11. M. V. Bhanderi, and H. B. Shah. "Machine Learning for Wireless Sensor Network: A Review, Challenges and Applications." Adv. Electron. Electr. Eng 4 India, Vol. 4, Number 5, pp. 475-486, 2014.

12. A. M. Bongale, A. Swarup, and S. Shivam. "EiP-LEACH: Energy influenced probability based LEACH protocol for Wireless Sensor Network." In 2017 International Conference on Emerging Trends \& Innovation in ICT (ICEI), IEEE, pp. 77-81, Feb 3-5, 2017.

13. M. Bidaki, and R. K. Tabbakh. "Efficient Fuzzy Logic-Based Clustering Algorithm for Wireless Sensor Networks." International Journal of Grid and Distributed Computing, Vol. 9, No. 5, pp.79-88, 2016.

14. M. Toloueiashtian and H. Motameni. "A new clustering approach in wireless sensor networks using fuzzy system." The Journal of Supercomputing, Vol. 74, no. 2, pp.717-737, 2017.

15. J. S. Pan, S. C. Chu, T. K. Dao, and V. C. Do. "Improved Performance of Wireless Sensor Network Based on Fuzzy Logic for Clustering Scheme." In International Conference on Smart Vehicular Technology, Transportation, Communication and Applications, Springer, pp. 104-113, Cham, 2019.

16. J. Wang, J. Niu, K. Wang, and W. Liu. “An energy efficient fuzzy cluster head selection algorithm for WSNs.” In 2018 International Workshop on Advanced Image Technology (IWAIT), IEEE, pp. 1-4, 2018.

17. H. AA Al-Kashoash, Z. SA Rahman, and E. Alhamdawee. "Energy and RSSI based fuzzy inference system for cluster head selection in wireless sensor networks." In Proceedings of the International Conference on Information and Communication Technology, pp. 102-105. ACM, 2019.

18. R. Zaghal, F. Alyounis, and S. Salah. "Performance evaluation of routing protocols in wireless sensor networks: A comparative study." In 5th international conference on informatics and applications (ICIA), pp. 63-70. 2016.

19. N. Sanadhya, "A Comparative Study on Routing Protocols Used in LEACH", International Journal of Engineering Development and Research, Vol. 3, pp.482-486, 2015.

20. J. Wang, J. Niu, K. Wang, and W. Liu. “An energy efficient fuzzy cluster head selection algorithm for WSNs.” In 2018 International Workshop on Advanced Image Technology (IWAIT), IEEE, pp. 1-4., 2018.

21. S. S. Barani and S. U. Visalakshi, "Super Cluster Head Selection and Energy Efficient Round Robin Load Balancing Technique in Wireless Sensor Networks", International Journal of Engineering Science and Computing,Vol.7, No.4, pp.10065-10072, April 2017.

22. A. S. Toor, and A. K. Jain. "A survey of routing protocols in Wireless Sensor Networks: Hierarchical routing.” In 2016 International Conference on Recent Advances and Innovations in Engineering (ICRAIE), IEEE, pp. 1-6, 2016.

23. M. Elshrkawey, S. M. Elsherif, and M. E. Wahed. "An enhancement approach for reducing the energy consumption in wireless sensor networks." Journal of King Saud University-Computer and Information Sciences, Vol 30, no.2, pp.259$267,2018$.

24. H. Oudani , S. Krit, M. Kabrane, K. Bandaoud, M. Elaskri, K. Karimi, H. Elbousty, and L. Elmaimouni. "Energy efficient in wireless sensor networks using cluster-based approach routing." International Journal of Sensors and Sensor Networks, Vol. 5, No. 5-1, 2017, pp. 6-12.

25. M. Tamene, and K. N. Rao , "Fuzzy C-Means Clustering Algorithm for Optimization of Routing Protocol in Wireless Sensor Networks", International Journal of Computer Science and Network (IJCSN), Vol.5, pp.2277-5420, April 2016.

26. R. M. Al-Kiyumi, C. H. Foh, S. Vural, P. Chatzimisios, and R. Tafazolli. "Fuzzy logic-based routing algorithm for lifetime enhancement in heterogeneous wireless sensor networks." IEEE Transactions on Green Communications and Networking, Vol. 2, no. 2:pp. 517-532, 2018.

27. M. Singh, and S. K. Soni. "A comprehensive review of fuzzy-based clustering techniques in wireless sensor networks." Sensor Review, Vol. 37, no. 3: pp.289-304, 2017. 